\title{
CORRIGENDUM
}

\section{Joint analysis of spatial genetic structure and inbreeding in a managed population of Scots pine}

\author{
MR García-Gil, F Olivier, S Kamruzzahan and P Waldmann
}

Heredity (2009) 103, 97; doi:10.1038/hdy.2009.60

Correction to: Heredity (2009) 103, 90-96; doi:10.1038/ hdy.2009.33

Since the publication, the authors have noticed that the name of the second author in the above paper was published incorrectly. The correct name of this author is O François.

We would like to apologize for any inconvenience caused. 